\title{
Bilateral ovarian metastases from ureteric urothelial cancer: Initial case report and distinguishing role of immunohistochemistry
}

\author{
Vivek Venkatramani, MD; ${ }^{*}$ John Samuel Banerji, MD; Ramani Manojkumar, $M D^{+}$
}

*Department of Urology, Christian Medical College, Vellore, India; 'Department of Pathology, Christian Medical College, Vellore

Cite as: Can Urol Assoc J 2015;9(1-2):E52-4. http://dx.doi.org/10.5489/cuaj.2374

Published online January 12, 2015.

\section{Abstract}

Urothelial cancers of the upper tract are aggressive malignancies with a propensity for distant metastases. Transitional cell carcinoma can also develop de novo in the ovaries and differentiation between these lesions requires immunohistochemistry. We report a case of right lower ureteric urothelial carcinoma with metastases to both ovaries. To our knowledge, this is the first reported case of bilateral ovarian metastases from an upper tract primary, diagnosed with immunohistochemistry.

\section{Introduction}

Urothelial cancers of the upper tract are aggressive malignancies with a propensity for distant metastases; however metastasis to the ovary has been reported only once. ${ }^{1}$ Transitional cell carcinoma (TCC) can also develop de novo in the ovaries, accounting for $1 \%$ to $2 \%$ of ovarian tumours. ${ }^{2}$ It can be difficult to distinguish these from metastatic lesions without the help of immunohistochemistry.

We report a case of right lower ureteric urothelial carcinoma, and a concomitant superficial bladder tumour with metastases to both ovaries. To our knowledge, this is the first reported case of bilateral ovarian metastases from an upper tract primary, diagnosed with immunohistochemistry.

\section{Case report}

A 56-year old female underwent right nephrectomy elsewhere after evaluation for hematuria revealed a non-functioning kidney with hydroureteronephrosis. Histopathology revealed chronic pyelonephritis with no evidence of malignancy.

Two months later she presented to us with persistent, painless, gross hematuria. Contrast-enhanced computed tomography revealed a thickening of the right lower ureteric stump with no periureteric stranding, and an enhancing lesion in the bladder (Fig. 1a, Fig. 1b). There were no significant regional lymphadenopathy or liver or lung metastases. The ovaries appeared normal.

At cystoscopy, a $2 \times 2$-cm papillary tumour in the region on the bladder trigone was noted, which was resected. No perforation occurred during resection. Retrograde ureterogram revealed a narrow ureteric stump, precluding ureteroscopy. The histopathology of the bladder tumour showed high-grade urothelial carcinoma pT1.

Four weeks later, she underwent a re-staging resection, and ureteroscopy revealed a papillary tumour, which was biopsied. The scar resection revealed no residual tumour and the ureteric biopsy showed carcinoma in situ. She was planned for open ureteric remnant and bladder cuff excision, however due to personal constraints she deferred the surgery for a month.

Intra-operatively the ureteric remnant appeared dilated and thickened. There was no obvious para-ureteric lymphadenopathy. Both ovaries were enlarged and replaced by solid masses and omental nodules were noted. Bilateral salpingoopherectomy and an infracolic omentectomy were performed, in addition to the completion ureterectomy and bladder cuff excision. The final histopathology revealed a ureter entirely involved by high-grade urothelial malignancy pT2.

Both ovaries were replaced by high-grade metastatic urothelial carcinoma (Fig. 2). Immunohistochemistry revealed positive staining for CK7 and CK20 (Fig. 3a, Fig. 3b), and negative staining for WT1. The omentum also showed multiple tumour deposits. She received 2 cycles of palliative chemotherapy with gemcitabine and cisplatin.

\section{Discussion}

To our knowledge only a single case of metastases associated with ureteric malignancy has been reported. ${ }^{1}$ This particular case had multiple urothelial tumours (left renal 


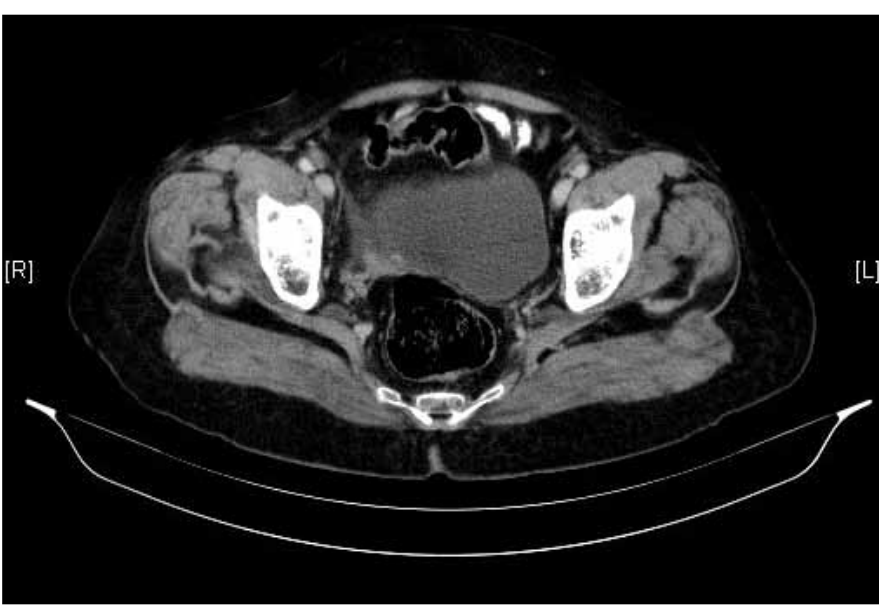

Fig. 1a. Venous phase of contrast-enhanced computed tomography showing thickening and enhancement of right lower ureter.

pelvis, left ureter, bladder and urethra), and other researchers have classified the renal pelvis as the primary site that led to metastases. ${ }^{1,3}$ To the best of our knowledge, this makes our case the first in which bilateral ovarian metastases from a primary ureteric malignancy have been identified. Our patient had a small bladder tumour as well, but it was nonmuscle invasive and there was no perforation during the resection. Re-staging transurethral resection of the bladder tumour showed no residual tumour, indicating that the ureteric primary was responsible for metastasis.

Metastases to the ovary account for about $6 \%$ of ovarian malignancies. ${ }^{4} \mathrm{~A}$ "renal-ovarian" axis has been proposed to account for the metastatic spread to the ovaries. ${ }^{3}$ An incompetent left gonadal vein, which allows retrograde flow, has been proposed as the possible mechanism. ${ }^{3}$ This is supported by the predominance of left-sided primary lesions in the literature. ${ }^{3}$

Delay in diagnosis is another important factor in metastasis to the ovary, contributing to $38 \%$ of cases in a study. ${ }^{3}$ Our patient had undergone a right nephrectomy without further evaluation of the cause of hydroureteronephrosis or hematuria. There was no malignancy in the renal histopathology making spread from the kidney unlikely. She presented to us late, and subsequently delayed excision of the ureteric stump by a month.

Diagnosis is difficult because primary TCC can arise in the ovary as well. Primary TCC of the ovary is more likely to have mucin pools, and thick papillae with smooth luminal borders, while pseudo-papillae secondary to cell necrosis are more common in metastatic lesions. ${ }^{3,4}$ Immunohistochemical analysis has greatly simplified this situation. Primary ovarian TCC expresses markers similar to other ovarian epithelial malignancies. ${ }^{5}$ It stains positive for vimentin, CA-125 and Wilm's tumour protein (WT1), and may express CK7. Metastases from the urinary tract express thrombomodulin, uroplakin III, CK7 and CK20. ${ }^{5}$ The combination of CK7 and

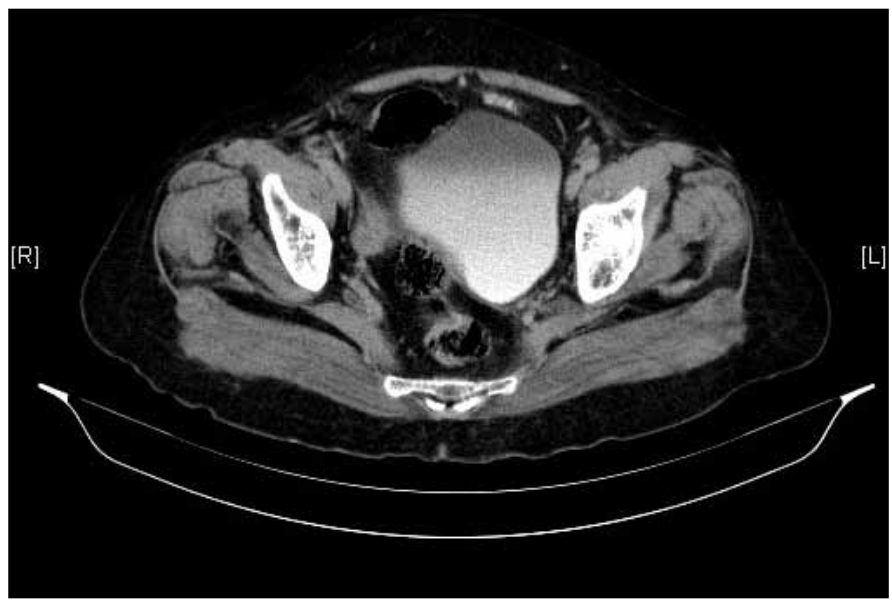

Fig. 1b. Delayed phase of contrast-enhanced computed tomography with thickening of the lower ureter.

CK20 expression is specific for urinary tract origin. ${ }^{5}$ In our case, the ovarian lesions were positive for CK7 and CK20, and negative for WT1, indicating the primary was in the urinary tract. Differentiation is important as ovarian primaries are more chemosensitive and have a better prognosis. ${ }^{5}$

\section{Conclusion}

To our knowledge, our case represents the first documented case of bilateral ovarian metastases secondary to a primary ureteric malignancy. Immunohistochemistry allows ready differentiation of metastases from primary ovarian TCC.

Competing interests: Authors declare no competing financial or personal interests.

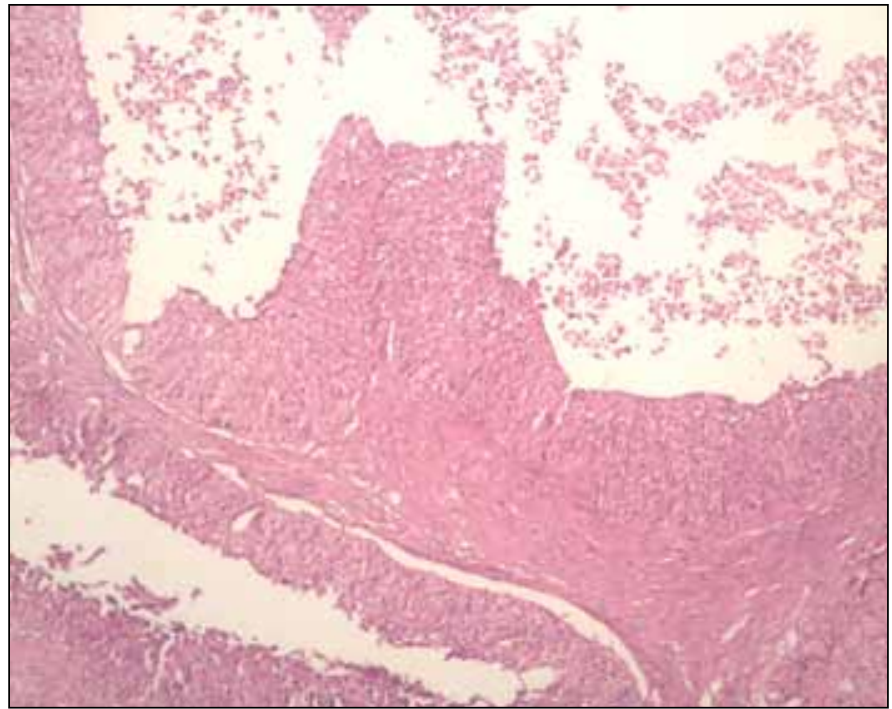

Fig. 2. Section of the ovary with metastatic urothelial carcinoma (hematoxylin \& eosin stain $\times 100$ ) 
Venkatramani et al.

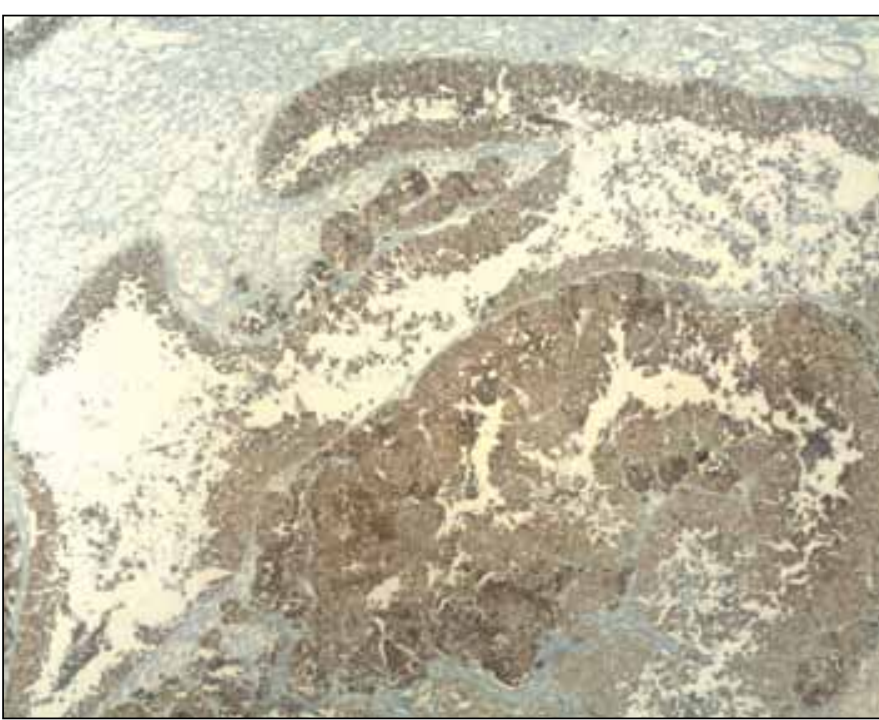

Fig. 3a. Section of ovary with positive CK-20 staining (CK-20 IHC, 50×).

This paper has been peer-reviewed.

\section{References}

1. Fosså SD, Schiolseth SA, Miller A. Multiple urothelial tumours with metastases to uterus and left ovary. A case report. Scand J Urol Nephrol 1977;11:81-4. http://dx.doi.org/10.3109/00365597709179697

2. Young RH, Scully RE. Urothelial and ovarian carcinomas of identical cell types: Problems in interpretation. A report of three cases and review of the literature. Int J Gynecol Pathol 1988;7:197-211. http://dx.doi. org/10.1097/00004347-198809000-00001

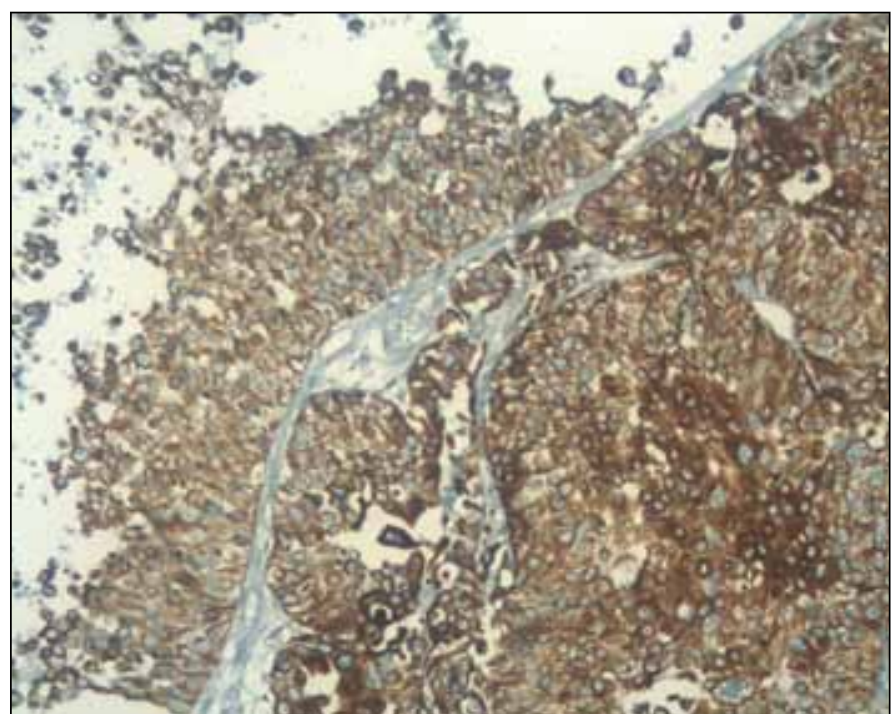

Fig. 3b. Higher power view of ovary with positive CK-20 staining (CK-20 IHC, 200x).

3. Gavallos $G$, Tawfik 0 , Herrell D, et al. Renal-ovarian axis: A case report and review. Urology 2003;62:749. http://dx.doi.org/10.1016/S0090-4295(03)00585-5

4. Eichhorn JH, Young RH. Transitional cell carcinoma of the ovary: A morphologic study of 100 cases with emphasis on differential diagnosis. Am J Surg Pathol 2004;28:453-63. http://dx.doi. org/10.1097/00000478-200404000-00004

5. Tazi EM, Lalya I, Tazi MF, et al. Transitional cell carcinoma of the ovary: A rare case and review of literature. World J Surg Oncol 2010;8:98. http://dx.doi.org/10.1186/1477-7819-8-98

Correspondence: Dr. Vivek Venkatramani, Department of Urology, Christian Medical College, Vellore, India; docvivek@gmail.com 